REVISTA ANDALUZA DE ANTROPOLOGÍA.

NÚMERO 10: ANTROPOLOGÍA Y EPISTEMOLOGÍAS DEL SUR: EL RETO DE LA DESCOLONIZACIÓN DE LA PRODUCCIÓN DEL CONOCIMIENTO

MARZO DE 2016

ISSN 2174-6796

[pp. 54-78]

http://dx.doi.org/10.12795/RAA.2016.10.04

\title{
PARADIGMA DE INVESTIGACIÓN Y PUEBLOS INDÍGENAS DEL PRIMER MUNDO: CRÍTICA CULTURAL DEL TRABAJO SOCIAL
}

\section{RESEARCH PARADIGM AND INDIGENOUS PEOPLES OF THE FIRST WORLD: A CULTURAL CRITIQUE OF SOCIAL WORK}

\section{Kepa Fernandez de Larrinoa}

\section{Grupo de Investigación Lera-Ikergunea}

Departamento de Trabajo Social. Universidad Pública de Navarra

\section{Resumen.}

Este ensayo aborda varios asuntos. De un lado, el ejercicio profesional del trabajo social en comunidades indígenas. De otro, la creación y transmisión de conocimiento al respecto en centros de investigación y educación superior. Con tal fin, se presentan las propuestas de trabajo social indígena formuladas por docentes e investigadores indígenas adscritos a departamentos de trabajo social en universidades de países anglosajones económicamente opulentos y con población nativa en su seno. Son Australia, Nueva Zelanda, Canadá y Estados Unidos. Particularmente, se discuten aspectos teóricoepistemológicos afines al campo de los estudios culturales, poniendo atención en las nociones de la poscolonialidad y subalternidad. En línea con lo anterior, se explica que tres principios resultan hoy insoslayables en la reflexión sobre el trabajo social indígena: uno es el concepto de competencia cultural; otro, la noción de indigenización del trabajo 
social; y tercero, qué se entiende en el trabajo social indígena por metodologías y técnicas de intervención social descolonizantes. Consecuentemente, en este artículo se examina la noción de paradigma de investigación indígena, que se analiza según su formulación en el contexto histórico y cultural de dominación de las poblaciones nativas del primer mundo. Del conjunto argumental resulta una crítica cultural de los saberes universitarios y prácticas profesionales vinculadas al trabajo social.

\title{
Palabras clave.
}

Crítica cultural; trabajo social; competencia cultural; paradigma de investigación e indígena.

\begin{abstract}
.
This paper addresses the practice of social work in indigenous communities as well as creating and transmitting social work knowledge in research centres and higher education institutions. It focuses on how social intervention has been analysed by indigenous lecturers and researchers who are attached to social science and humanities departments at universities in economically affluent English-speaking countries with indigenous population, such as Australia, New Zealand, Canada and the United States.

To that end the paper, first, examines theoretical and epistemological issues related to the field of cultural studies. Second, it pays attention to the notions of subordination and postcoloniality. Third, it is argued that three principles are unavoidable today when reflecting on indigenous social work: one is the concept of cultural competence; another, the notion of indigenization of social work; and next, what is meant by decolonizing research methodologies and techniques as indigenous peoples explain them. Thus, this article approaches the notion of indigenous research paradigm, which is explored by looking at how native scholars from First World countries make sense of the historical and cultural context of domination under which their communities still remain. Finally, the article develops a cultural critique of university knowledge and professional practices linked to social work.
\end{abstract}

\section{Key words.}

Cultural critique; social work; cultural competence; indigenous research paradigm. 


\section{INTRODUCCIÓN ${ }^{1}$}

Este artículo versa sobre el nexo entre conocimiento universitario, epistemología científica e intervención sociocultural en el seno de las poblaciones indígenas. Lo hace a partir de la reflexión al respecto por parte de un nutrido grupo de docentes e investigadores universitarios de origen nativo. Se centra en la noción de trabajo social indígena y expone el tipo de propuestas de intervención social asociadas a dicha noción según las vienen últimamente formulando los intelectuales y activistas comunitarios indígenas adscritos a departamentos de trabajo social. Concretamente, departamentos de trabajo social en universidades anglosajonas de países opulentos. Países ricos con población aborigen entre sus ciudadanos nacionales son Australia, Nueva Zelanda, Canadá y Estados Unidos. De acuerdo con lo anterior, la discusión que aquí se presenta se ciñe a la relación entre reflexión teórica y acción sociocultural comunitaria según la experiencia histórico-cultural y política de la población indígena residente en el Primer Mundo.

Por otro lado, en el artículo se pone especial atención en las propuestas teóricoepistemológicas y metodológicas que, llegadas desde el campo de los estudios culturales, se presentan a sí mismas en términos de descolonización y emancipación indígena. Son propuestas desde las que se defiende la idoneidad de generar curricula universitarios en trabajo social acordes con el decir y pensar de las poblaciones indígenas sobre sus necesidades perentorias. De sus dificultades teóricas y prácticas se ocupa este ensayo.

Finalmente, en este artículo se explica que un programa curricular de trabajo social indígena requiere un análisis previo de los límites y alcance de estos conceptos: competencia cultural; indigenización del trabajo social; metodologías de intervención social descolonizantes. Dicho análisis destapa un hecho significativo: los saberes universitarios no son per se universales y objetivos, sino que forman parte del atrezzo teatral de la modernidad occidental.

1. Este artículo está vinculado a la investigación de título "Modelos y narrativas en la intervención sociocultural y desarrollo comunitario indígena", que llevé a cabo en el Instituto de Antropología Social de la Universidad de Oxford y Wolfson College durante 11 meses el curso 2011-2012. Agradezco a los profesores Jeremy MacClancy y Marcus Banks su tiempo, ayuda y consejo. Asimismo, agradezco a Professor Dame Hermione Lee, presidente de Wolfson College, el privilegio de haber sido parte de la institución que dirige. También, agradezco los comentarios y sugerencias formuladas sobre el texto por los o las evaluadoras anónimas encargadas de revisarlo. 


\section{TRABAJO SOCIAL INDÍGENA ${ }^{2}$}

Hay modelos de trabajo social que, en simbiosis con el conocimiento antropológico universitario, propician el diagnóstico de necesidades sociales -e igualmente el diseño de proyectos de intervención social y su ejecución- desde el diálogo con el método etnográfico. La intervención social con poblaciones nativas es un lugar en que el trabajo social y la antropología han encontrado un espacio específico de interlocución mutua.

Asimismo, trabajo social y antropología son campos de acción sociocultural que tienden a entrecruzarse al examinar el tipo de praxis social que acompaña a la conjunción de las nociones de comunidad y desarrollo (Cole, 1977; Cohen, 1985; Kupper, 1992; Wright, 1992; Dominelli, 2007; Rahnema, 1977; Hobart, 1993; Escobar, 1995). Esta conjunción teórico-práctica ha delimitado un espacio interdisciplinar para la práctica y la reflexión de la acción social colectiva: el desarrollo cultural sociocomunitario, donde confluyen, como decimos, el trabajo social, la educación social, la animación sociocultural, las artes comunitarias, la antropología aplicada y otras disciplinas (Abatena, 1995; Payne, 1991; Taylor y Roberts, 1985; Giroux, 1997, 2001; Valverde-Resalt, 1997; Bartoli, 2002; Sánchez, 2003; Caride y Vieites, 2006; van Erven 2013; McDonald, 2002; Pink, 2006).

El desarrollo cultural y sociocomunitario indígena es un campo de indagación crítico de la cultura y las sociedades occidentales y modernas. Entre las razones que lo explican destacan: su consistencia con la realidad indígena contemporánea y sus procesos de fortalecimiento interno; su énfasis en la acción social y el desarrollo cultural comunitario colaborativo y participativo; y su labor de impulso de metodologías y técnicas de investigación acción (Dixon y Scheurell, 1995; Gray, Coates y Yellow Bird, 2008; Kothari, 1999; Verhelst y Tyndale, 2002; Harrison, 2001; Saugestad, 2001; Sillitoe, Bicker y Pottier, 2002; McTaggart, 1997; Craig y Mayo, 1995).

Se advierte, pues, que la ecuación trabajo social/antropología cultural ha provocado una interacción dialéctica intensa entre, de un lado, quienes estudian las lógicas de funcionamiento interno características de las distintas comunidades socioculturales humanas y, de otro, quienes estudian el cambio sociocultural planificado e inducido desde los aparatos gubernamentales y sus instituciones nacionales e internacionales. $\mathrm{Y}$ ocurre que hay, cuando menos, tres ámbitos de indagación dialogando entre sí: el de los estudios antropológicos de comunidad; el de los estudios de antropología aplicada al desarrollo; y el de los estudios de trabajo social sociocomunitario (James, Nadarajah, Haive y Stead, 2012).

Las categorías "indio", "nativo" y "aborigen" se asientan en situaciones de colonialidad. Sin embargo, el trabajo social emerge en procesos de modernización. Significativamente,

2. En la exposición que sigue se citan fragmentos de obras consultadas en lengua inglesa. Cuando así ocurre, yo mismo las he traducido al castellano e incorporado al texto principal. 
cuando analizamos la praxis colonial vis à vis la retórica de la modernidad descubrimos que indio, nativo y aborigen son categorías surgidas en situaciones de dominación política, económica y cultural, situaciones en que las metrópolis dominantes proyectan sobre las poblaciones locales criterios de clasificación étnico-raciales, que al objetivar su humanidad congelan su historicidad, anulando así su subjetividad. Un efecto de la colonialidad es que eso que denominamos "identidad indígena" es una identidad subalterna. De ahí la importancia de teorizar sobre las propuestas y experiencias de intervención/acción orientadas a la descolonización del pensamiento y las prácticas socioculturales (Wolf, 1987; Bonfil, 1987; Ashcroft, Griffiths y Tiffin, 1995; Quijano, 2000; Mignolo, 2007; Mezzadra, 2008).

Que el trabajo social se ha internacionalizado y globalizado es un hecho evidente: desde los barrios de las ciudades se ha desplazado a los pueblos rurales de Europa; ha viajado hasta los países del Sur; y ahora penetra en las comunidades indígenas. De suyo, el trabajo social es una profesión de raíces urbanas, preocupada por los efectos negativos de la Revolución Industrial en la sociedad y los individuos, esto es, interesada en la resolución de los desajustes psicológicos y socio-estructurales que el salto de la sociedad tradicional a la modernidad ha causado en algunos individuos, familias y grupos humanos. Siendo el trabajo social una consecuencia de la historia social europea más inmediata, surge la duda de hasta qué punto los ingredientes europeos del trabajo social son un lastre o una prestación positiva en situaciones donde la intervención social va dirigida a solucionar problemas individuales y sociales de carácter indígena.

Un libro en que sus autores reflexionan sobre lo anterior es el de Mel Gray, John Coates y Michael Yellow Bird (2008), quienes se preguntan cuánto de cierto hay en la afirmación de que el trabajo social es una invención occidental asida a varias características de los Estados-nación, como son, entre otras, la idea de modernidad e individualismo, una economía productivo-industrial y las formaciones políticas demócrata-cristianas y social-demócratas. Igualmente, explican que la internacionalización académica del trabajo social y la globalización de su práctica durante los últimos años son efecto de la modernidad tardía, esto es, del tardocapitalismo económico. Dicho de otro modo, la expansión económica mundial viene acompañada de otra expansión, la del trabajo social. Pero ocurre que la internacionalización del trabajo social y su implantación en países del Sur acarrean un peligro, el del imperialismo o colonización profesional. Y es que, al igual que otras profesiones modernas occidentales, el trabajo social se ha incorporado al memorandum de la globalización, y en su empeño ha adoptado una posición aglutinante y universalizante de la vida social, una que favorece intervenciones sociales similares en situaciones y contextos culturales disímiles (Truell, 2012).

Sobre la universalización del pensamiento y tipos de intervención social procedentes de la experiencia histórica del trabajo social occidental, James Midgley, crítico adelantado 
de la praxis y concepción intelectual del trabajo social internacional en países del Sur, se pronunció con el enunciado "imperialismo profesional del trabajo social en el Tercer Mundo" (Midgley, 1981). Frente a esta impugnación brutal del trabajo social fuera de Occidente, Mel Gray, John Coates y Michael Yellow Bird exhortan a que el trabajo social en comunidades indígenas sea consistente con el tipo de sociedad y cultura del lugar. De hecho, recelan del valor que IASSW y IFSW (las reputadas asociaciones internacionales de trabajo social International Association of Schools of Social Work e International Federation of Social Workers), últimamente han dado a la necesidad de registrar una definición de trabajo social global así como elaborar un memorándum global.

"El verdadero problema con que nos enfrentamos es el desarrollo en todo el mundo de una práctica y educación del trabajo social culturalmente relevante [...] Las proclamas en pro de un trabajo social 'global' y 'universal' presentan una paradoja a los interesados en la capacidad de respuesta del trabajo social a los contextos culturales locales [...] La mayoría de quienes están involucrados en trabajo social internacional están principalmente preocupados por el desarrollo de modelos de práctica del trabajo social culturalmente relevantes en contextos locales, entre culturas con diferentes grados de diferencia con respecto a la suya propia [...] Por lo tanto creemos que pretender un trabajo social 'global' no sólo es una exageración del alcance del trabajo social, sino que también niega la importancia de las organizaciones no gubernamentales y del desarrollo comunitario y social, que desempeñan un papel mucho más importante en el trabajo con la mayoría de la población mundial en estado de necesidad" (Gray; Coates y Yellow Bird 2008: xxv).

[Versión original: "The real issue with which we are dealing is the development of culturally relevant social work practice and education around the world ... Claims to 'global' and 'universal' social work present a paradox for those concerned with social work's responsiveness to local cultural contexts [...] Most who are involved in international social work are mainly concerned with developing models of culturally relevant social work practice in local contexts, among cultures with varying degrees of difference from their own [...]. Thus we believe that claims to 'global' social work are not only an exaggeration of the reach of social work but also deny the importance of nongovernment organizations, community and social development which play a far greater role in working with the majority of the world's population who are in need"].

Dos corolarios siguen del razonamiento anterior. Uno apunta hacia una "construcción de inocencia" dentro del trabajo social. Los críticos de la historia del trabajo social con comunidades indígenas denuncian la figura del trabajo social con apariencia inocente. Explican que esta imagen inocente del trabajo social es consecuencia de un proceso de naturalización social de la existencia y promoción de la profesión. Consiste en desplegar una imagen pública de la profesión tácitamente aferrada a las nociones de bondad, empatía y solidaridad humana, juzgándose su ejercicio como un asunto personal, 
intrínsecamente generoso y vocacional. Porque quienes se dedican a ello lo hacen de buen grado y colmados de buenas intenciones, el trabajo social es bueno por sí mismo, además de socialmente necesario. Sin embargo, cuando tanto la práctica como la ideología de la buena voluntad y el deseo de ayudar se desarrollan incrustados en el seno de las relaciones coloniales de dominación, la bondad, la empatía y la solidaridad humanas no pueden sino volverse perniciosas. Así ha sucedido en el caso de las sociedades y poblaciones indígenas (Clark et al., 2012: 113-114).

El segundo corolario apunta a que el conocimiento antropológico de las sociedades y culturas locales es condición previa a la intervención social. Los antropólogos insisten en que la práctica del trabajo social en comunidades indígenas debe estar afianzada en el concepto de cultura local, lo que quiere decir que la contribución de la antropología al trabajo social radica en el estudio de las culturas y sociedades locales dentro de un marco de investigación/acción etnográfica e intervención participativa (Tacchi, Slater y Hearn, 2003). Ahora bien, las comunidades indígenas actuales son el resultado de un largo proceso de colonización y opresión social, política, económica y cultural, a cuyo afianzamiento histórico los estudios de antropología sociocultural han contribuido con indudable desfachatez (Asad, 1973; Stocking, 1974; Lévi-Strauss, 1996).

Sin duda, la historia de los pueblos indígenas es una historia cargada de violencia, puesto que es la historia del despojo de sus tierras, cultura, dignidad y humanidad (Harper, 1986; Ferro, 2005). Por ello, el trabajo social en comunidades indígenas se vincula a la defensa de los derechos humanos y la participación activa en procesos locales de descolonización y de reapropiación cultural, social, política y económica (Weaver, 1999; Delaney y Brownlee, 1999). El trabajo social indígena contemporáneo no puede ser más explícito en lo concerniente con lo anterior, y es que en defensa de sus reclamaciones se apoya en la "Declaración Universal de los Derechos de los Pueblos Indígenas de Naciones Unidas” (Anaya, 1996; Álvarez, Oliva y Zúñiga, 2009).

Empero, perdura el recelo intelectual indígena hacia el campo de la ciencia e investigación disciplinaria occidentales, particularmente en lo que concierne al modo en que estas: bien contribuyen al desarrollo y bienestar efectivo de quienes forman parte de las comunidades culturales nativas; bien lo inhabilitan; bien se desentienden de él. La persistencia de esta desconfianza alcanza a los estudios contemporáneos de crítica cultural aludidos arriba, ampliándose a la generalidad del campo de estudios culturales poscoloniales, que gloso más abajo. Muestra cualificada de lo anterior lo proporciona Linda Tuhiwai Smith, profesora universitaria maorí de educación indígena, quien se ha preguntado hasta qué punto los estudios poscoloniales de crítica cultural no responden sino a una actualización del ánimo, quehacer y preocupación etnocentristas de Occidente para con las gentes al otro lado del jardín de la casa. Esto es, para con los indios. Pone el ejemplo de la poetisa Bobbi Sykes (1943-2010), cuando en una conferencia sobre poscolonialismo exclamó: 
“Qué? ¿Poscolonialismo? ¿Se han marchado?” (en Smith, 1999: 24). Primera mujer negra australiana graduada en una universidad americana, en Australia Bobbi Skyes fue una activa defensora de los derechos de las poblaciones indígenas y sus mujeres, habiendo escrito, entre otras obras, la biografía una mujer especial, Shirley Colleen Smith (19241998), indígena australiana dedicada al trabajo social comunitario (Mum Shirl, 1981).

\section{CRÍTICA CULTURAL DEL TRABAJO SOCIAL}

El trabajo social indígena bajo el orbe anglosajón es resultado de la corriente reflexiva contemporánea gestada en el ámbito de los estudios culturales (During, 1993). Sobre una plataforma epistemológica ecléctica (Wuthnow et al., 1984; Austin-Broos, 1987), este campo de indagación originalmente aglutinó a un amplio elenco de autores llegados desde varios campos escolásticos, normalmente de lengua inglesa (Terdiman, 1985; Said, 1993; Hooks, 2000; Marcus y Fischer, 1986; Hall, 1997; Luszczynska, 2012; Jameson, 1991). Distintivo en los estudios culturales es su firmeza en revelar los transcursos históricos de dominación política y económica insertos en los procesos de construcción simbólica de identidades social y culturalmente subordinadas.

Personalmente postulo que es en este espacio académico occidental de reflexión crítica glosado arriba donde hoy mejores frutos puede dar la interlocución entre el trabajo social y la antropología cultural. Con todo, encuentro que esta necesidad de interlocución trabajo social/antropología en los términos recién expresados es todavía más sentida en el ejercicio -todavía inacabado- de repensar la teoría y práctica del trabajo social en el seno de las comunidades indígenas. Lo detallo en las páginas que siguen.

El área de conocimiento de los estudios culturales incluye dos esferas de indagación, los estudios poscoloniales y los estudios de subalternidad (Ashcroft, Grifiths y Tiffin, 1995). Es aquí, como digo, donde considero oportuno demarcar cualquier investigación actual sobre los tipos de proceder en el campo de la intervención social con comunidades indígenas. Es así que en las universidades de los países anglófonos opulentos se han abierto tres escenarios conceptuales relacionadas con la enseñanza teórica y práctica del trabajo social en comunidades indígenas: uno conocido con el epígrafe de colonialidad del conocimiento científico (Quijano, 2000); otro, como indigenización del trabajo social (Grey, Coates y Yellow Bird, 2010); y el tercero, en términos de acción social comunitaria descolonizante y emancipante (Smith, 2008; Gray, Coates y Hetherington, 2011).

Según se formula desde los estudios de crítica cultural, el trabajo social indígena es un sedimento postrimero en la historia estratigráfica del imperialismo occidental. De ahí que en sus escritos Linda Tuhiwai Smith subraye que el imperialismo, además de conjugar fuerzas económicas, políticas y militares, aglutine también fuerzas ideológicas complejas vinculadas a modos de expresión técnico, intelectual y cultural específicos. 
Entiende ella que el imperialismo es un proceso en el que confluyen asuntos como la expansión económica, la subyugación de la otredad y la producción y reproducción de conocimientos discursivos. Resultado de ello es que imperialismo, investigación, universidad y conocimiento son asuntos de transcurso entrelazado:

"Este punto de vista sobre el imperialismo lo localiza dentro del espíritu de la Ilustración, que marcó la transformación de la vida económica, política y cultural en Europa. En este amplio contexto de la Ilustración, el imperialismo se convierte en parte integral del desarrollo del Estado moderno, la ciencia, las ideas y la persona humana 'moderna'. En formas complejas, el imperialismo fue también un modo mediante el que los nuevos Estados de Europa pudieron expandir su economía, mediante el que nuevas ideas y descubrimientos pudieron realizarse y sujetarse, y mediante el que los europeos pudieron desarrollar su sentido de europeidad. La imaginación imperial permitió a las naciones europeas imaginar la posibilidad de que nuevos mundos, nuevas riquezas y nuevas posesiones existiesen, que podían ser descubiertas y controladas. Esta imaginación se realizó a través de la promoción de la ciencia, la expansión económica y la práctica política.

[...] Hay, por ejemplo, una mayor y más inmediata necesidad de entender las complejas formas en que las personas fueron introducidas dentro del sistema imperial, porque todavía se siente su impacto, a pesar de la aparente independencia adquirida por los antiguos territorios coloniales. La entrada del imperialismo en 'nuestras cabezas' reta a aquellos que pertenecen a comunidades colonizadas a entender cómo es que esto ocurrió, en parte, porque percibimos la necesidad de descolonizar nuestras mentes, de recuperarnos a nosotros mismos, de reclamar un espacio en el que desarrollar un sentido de auténtica humanidad. Este análisis del imperialismo ha sido referido más recientemente en términos de 'discurso poscolonial', el 'imperio escribe la vuelta' y/o 'escribiendo desde los márgenes'. Sin embargo, hay un cuerpo de escritura más político que se extiende al trabajo revolucionario, anticolonial de varios activistas (de los que sólo algunos, como Franz Fanon, en realidad escribió sus ideas), que también se basa en el trabajo de escritores negros y afroamericanos y otros escritores minoritarios cuyo trabajo ha surgido de una preocupación por los derechos humanos y civiles, los derechos de las mujeres y otras formas de opresión" (Smith, 1999: 22-23).

[Versión original: "This view of imperialism locates it in within the Enlightenment spirit which signalled the transformation of economic, political and cultural life in Europe. In this wider Enlightenment context, imperialism becomes an integral part of the development of the modern state, of science, of ideas and of the 'modern' human person. In complex ways imperialism was also a mode through which Europeans could develop their sense of Europeanness. The imperial imagination enabled European nations to imagine the possibility that new worlds, new wealth and new possessions existed that could be discovered 
and controlled. This imagination was realized through the promotion of science, economic expansion and political practice.

[...] There is, for example, a greater and more immediate need to understand the complex ways in which people were brought within the imperial system, because its impact is still being felt, despite the apparent independence gained by former colonial territories. The reach of imperialism into 'our heads' challenges those who belong to colonized communities to understand how this occurred, partly because we perceive a need to decolonize our minds, to recover ourselves, to claim a space in which to develop a sense of authentic humanity. The analysis of imperialism has been referred to more recently in terms such as 'post-colonial discourse', the 'empire writes back' and/or 'writing from the margins'. There is a more political body of writing, however, which extends to the revolutionary, anticolonial work of various activists (only some of whom, such as Franz Fanon, actually wrote their ideas down) that draws also upon the work of black and African American writers and other minority writers whose work has emerged out of a concern for human and civil rights, the rights of women and other forms of oppression"].

Linda Tuhiwai Smith delata, pues, que las explicaciones y raciocinios recogidos en los compendios de conocimiento científico elaborados en universidades e institutos de investigación no dejan de ser una forma interesada de explicar la realidad humana. Desde el punto de vista indígena, la ciencia y sus saberes parecen conformar un tipo de representación de la realidad estrechamente vinculado tanto a la génesis y desarrollo del colonialismo económico como al pensamiento imperial de las metrópolis en que aquel se apuntala.

\section{SISTEMA CULTURAL INDÍGENA Y TRABAJO SOCIAL}

Una característica de los pueblos indígenas en los años 2000 es su identificación con las nociones de biodiversidad y multiculturalidad (Fernández de Larrinoa, 2014: 279375). Consecuencia de lo anterior es que las instituciones occidentales encargadas del cuidado, creación, enriquecimiento y transmisión del saber científico se muestran hoy más proclives al reconocimiento del valor epistemológico de los conocimientos propios de las comunidades indígenas.

Se dan circunstancias como que muchos pueblos indígenas residen en ecosistemas sui géneris. También son singulares las lenguas que hablan y las lógicas culturales con que se desenvuelven. Y se advierten preocupaciones como el calentamiento global y el cambio climático, que otorgan a la investigación en ciencia ecológica, por poner un ejemplo, un brío extraordinario. Circunstancias como estas estimulan la incorporación de determinados tipos de conocimiento indígena a las investigaciones de los científicos occidentales. Ello hasta el punto de haberse acuñado en el campo de la ecología y los 
ecosistemas terrestres una nueva expresión: conocimiento ecológico tradicional (Menzies, 2006; Huntington, 2000), que de hecho ya forma parte del léxico administrativo de las instituciones gubernamentales y asociaciones privadas encargadas de gestionar los asuntos medioambientales. Pero, según se advierte, se trata de una aceptación más declamatoria que efectiva.

Siconocimientoecológicotradicionaleslaexpresión conqueloscientíficosmedioambientales admiten en sus disertaciones segmentos de conocimiento de poblaciones nativas específicas, competencia cultural es la expresión utilizada por los trabajadores sociales con el fin de integrar los conocimientos locales indígenas en sus proyectos de intervención. Sin embargo, al igual que ocurre con la propuesta de conocimiento ecológico tradicional, la noción de competencia cultural es un asunto generador de recelos tanto dentro de la enseñanza universitaria del trabajo social como en su ejercicio profesional. Como en el caso anterior, se trata de una aceptación más declamatoria que efectiva. Y es que el valor metodológico e epistemológico de la lógica cultural de los sistemas interpretativos con que operan las distintas agrupaciones sociales indígenas, en su interacción con el mundo externo y dentro de sí, es un asunto todavía en discusión y objeto de controversia (Peat, 2012; Hendry, 2014).

Por ejemplo, en su estudio de 1999 sobre el concepto de competencia cultural en la práctica profesional y enseñanza del trabajo social en Estados Unidos, Hilary N. Weaver, profesora e investigadora de origen lakota, concluyó:

"No he sido capaz de identificar ningún trabajo empírico sobre competencia cultural con nativos norteamericanos [...].

Del mismo modo, es difícil estimar el número de trabajadores sociales norteamericanos nativos. Con la excepción de la Asociación Indio-Americana de Educadores de Trabajo Social (aproximadamente 30 miembros activos), no hay asociación nacional alguna de trabajadores sociales nativoamericanos en funcionamiento. Sin que la capacitación de trabajadores sociales indígenas para la provisión de atención y ayuda en sus comunidades de origen deje de ser un asunto crucial, todos los trabajadores sociales deben ser responsables de la provisión de servicios culturalmente competentes" (Weaver, 1999: 218-219).

[Versión original: "I have not been able to identify any empirical work on cultural competence with Native Americans [...].

Likewise, it is difficult to estimate the number of Native American social workers. With the exception of the American Indian Social Work Educators' Association (active membership approximately 30), there is no functioning national association of Native American social workers. Although it is critical to train indigenous social workers to provide care in their home communities, all social workers must be accountable for providing culturally competent services"]. 
En 2012, la revista Native Social Work Journal, editada por Lauretian University, Ontario, publicó un artículo examinando el grado de incorporación de los modelos culturales y sistemas de conocimiento e interacción social indígenas en los planes de estudio de las Escuelas Universitarias de Trabajo Social operantes en India y Canadá. Se afirmaba como sigue:

"Examinar el marco ético de nuestra investigación según se desarrolla requiere basarnos en el contexto sociopolítico del trabajo social en Canadá e India. La conciliación de la teoría y las prácticas del trabajo social con los pueblos indígenas de Canadá se encuentra actualmente en diálogo crítico, lo que se debe a la contribución histórica, todavía duradera, de la profesión del trabajo social a la colonización (...) Del mismo modo, en India, y en general en todo el mundo, 'los servicios humanos han contribuido a prácticas de colonización y desposesión' (...).

Aunque se ha conseguido cierto equilibrio a través de currículos innovadores centrados en lo indígena y espacios universitarios seguros en que el trabajo social se transmite a la siguiente generación de estudiantes, las contribuciones en el campo de la educación siguen siendo escasas. Como sobresale en los relatos de nuestra investigación original, (...), se siente la necesidad de que el campo de la educación sea un fuerte recinto de teorías y prácticas de trabajo social indígena. Es el espacio donde la instrucción curricular o teoría se conjunta con el aprendizaje experimental y la identidad de un trabajador social (...)" (Clark et al., 2012: 115-116).

[Versión original: "Examining the ethical framework of our research as it unfolds also requires we ground ourselves in the sociopolitical context of social work in both Canada and India. The reconciliation of social work theory and practices with Indigenous peoples of Canada is currently in critical dialogue due to the social work profession's historic and ongoing contribution to colonization (...) Similarly, within India and more generally throughout the world 'the human services have contributed to the practices of colonization and dispossession' (...).

Although some footing has been made through innovative, Indigenous centred curriculum and university safe spaces where social work is transmitted to next generation of students, contributions in the field education remain scarce. As highlighted in stories from our original research development grant (...), the need for field education to be a strong site of Indigenous centered social work theories and practices is necessary. It is the space where curriculum instruction or theory comes together with experimental learning and the identity of a social worker"].

Se observa, pues, que la ausencia de un trabajo social estadounidense específicamente pensado en términos indígenas al que finalizando el siglo XX aludía Hilary N. Weaver se repite en Canadá trece años después, ya en el siglo XXI. No obstante estas carencias, sería 
del todo injustificado concluir una inexistencia de experiencias de trabajo social indígena ejecutadas por trabajadores sociales indígenas al tiempo que basadas en concepciones culturales indígenas. Muestra de lo contrario es el estudio que Robyn Munford y Jackie Sanders han expuesto acerca de las prácticas de trabajo social indígenas desarrollas por trabajadores sociales maorís en Aotearoa, Nueva Zelanda:

"De particular importancia para los maoríes, y central para el logro de bienestar, es la conexión con la ascendencia de uno mismo y la puesta en primer plano de los sistemas de significados culturales, incluyendo la espiritualidad (...). Las relaciones terapéuticas exitosas comienzan con una comprensión de la posición de un cliente dentro de su familia $y$ la comunidad, y de las conexiones de su familia, tanto históricas como actuales. Antes de que cualquier proceso de ayuda pueda iniciarse, hay que tomar un tiempo para hacer las conexiones con el lugar (de donde la gente viene y donde actualmente se encuentra) $y$ las personas (a las que están conectados y los lazos generacionales significativos). Estas conexiones proporcionan una sólida base sobre la que se construirá el trabajo. Sin la debida atención a estos aspectos de las relaciones, es poco probable que el trabajo constructivo llegue a tener éxito, ya que el cliente se sentirá aislado de los elementos contextuales y relacionales que contribuirán a abordar los desafíos y trabajar por el cambio" (Munford y Sanders, 2010: 3)

[...]El compromiso de los trabajadores sociales y de la comunidad en Nueva Zelanda a adoptar nuevas formas de trabajar y aceptar los retos de marcos alternativos es parte de una búsqueda de modelos que puedan apoyar de manera más efectiva a las familias y las comunidades (...). Un ejemplo notable de esto es el modelo de Te Whare Tapu Wha (...), que ha proporcionado una manera holística de entender la salud y el bienestar que ha creado un conocimiento práctico y político en los servicios humanos de base. Este modelo hace hincapié en el yo como un ser psicosocial con dimensiones espirituales, psicológicas, físicas $y$ de parentesco. Marcos de práctica como este se utilizan ahora ampliamente, y los modos de entendimiento social y las experiencias de salud maoríes nutren cada vez más el enfoque por parte del Estado y los organismos no gubernamentales (ONGs)" (Munford y Sanders, 2010: 9).

[Versión original: "Of particular importance for Maori, and central to achieving wellbeing, is the connection with one's ancestry and a foregrounding of cultural meaning systems, including spirituality (...). Successful therapeutic relationships begin with an understanding of a client's position within their family and community, and of their family connections both historical and current. Before any helping process can begin, time must be taken to make connections to both place (where people come from and where they are currently located) and to people (who they are connected to and the significant generacional links). These connections provide a strong foundation upon which work will be built. Without due attention to these aspects of relationships, building work is unlikely to be successful, as the 
client will feel isolated from the contextual and relational elements that will contribute to addressing challenges and working on change.

[...] The commitment of social and community workers in New Zealand to embrace new ways of working and to be challenged by alternative frameworks is part of a search for models that can more effectively support families and communities (...). A notable example of this is Te Whare Tapu Wha model (...) which has provided a holistic way of understanding health and wellbeing that has shaped practice and policy understandings across a board base of human services. This model emphasises the self as a psycho-social being with spiritual, psychological, physical and kinship dimensions. Frameworks of practice such as this are now used widely and Maori understandings of social and health experiences increasingly inform the approach to practice of state and non-government agencies (NGOs)"].

Los tres estudios glosados arriba corroboran la ambivalencia, cuando no fragilidad de planteamiento, que -como se observa- acompañan a las epistemologías y metodologías indígenas en el debate sobre la conexión entre conocimiento universitario, trabajo social y sistema cultural nativo. Para ilustrar la naturaleza de tal ambivalencia y/o fragilidad conviene distinguir entre estas dos expresiones: trabajo social culturalmente competente o sensible; $y$ trabajo social radical indígena. La primera se refiere al proceso tenso, dinámico y contradictorio que sigue de la propuesta de incorporar las distintas cosmovisiones, modos de conocimiento y modelos de interpretación de la realidad, que son propios de los sistemas de sociabilidad comunitarios indígenas, a la práctica profesional y enseñanza universitaria del trabajo social actuales, asuntos históricamente externos al sistema conceptual en que se basa la sociabilidad indígena. En cambio, la segunda expresión alude al proceso -asimismo, tenso, dinámico y contradictorio- de pensar y ejercitar el trabajo social desde perspectivas y metodologías estrictamente indias, esto es, desde la radicalidad conceptual y existencial indígena. Un profesor de trabajo social en la Universidad de Manitoba (Canadá) y nativo cree, Michael Anthony Hart lo explica como sigue:

"Las cosas están cambiando en el ámbito de la investigación. Mientras que una vez, nosotros, como pueblos indígenas, cuando entramos en el mundo académico nos vimos enfrentados a dejar nuestra indigeneidad en la puerta, ahora varios de nosotros estamos trabajando activamente para asegurar que nuestra investigación no sólo sea respetuosa o 'culturalmente sensible', sino que también se base en los enfoques y procesos que forman parte de nuestras culturas" (Hart, 2010: 1).

[Versión original: "Things are changing in the realm of research. While at one time, we, as Indigenous peoples, were faced with leaving our indigenity at the door when we entered the academic world, several of us are now actively working to ensure our research is not only respectful or 'culturally sensitive', but is also based in approaches and processes that are parts our cultures"]. 
Michael Anthony Hart resalta la existencia de una dualidad de planteamientos en lo que concierne a los conocimientos indígenas y a los conocimientos universitarios, ello según aparecen asociados entre sí y con el trabajo social llevado a cabo con poblaciones indígenas. De un lado, advierte la omnipresencia de una fuerza central de pensamiento o cosmovisión, eminentemente américo-europea, desde la que las demás corrientes de pensamiento son juzgadas, interpretadas, vislumbradas y canalizadas. De ahí que, el nexo entre interpretación indígena e interpretación universitaria del trabajo social aparezca, en el mejor de los casos, incorporado a la retórica administrativa del respecto y tolerancia de la diferencia cultural, característicamente recurrente en el imaginario multicultural de los países opulentos de habla inglesa. Ocurre en este caso que la competencia y sensibilidad culturales en la intervención social consisten en seleccionar aquellos fragmentos de conocimiento e interpretación de la realidad indígena que, tras recogerlos y aislarlos del sistema cultural al que pertenecen, mejor se ajusten al engranaje del sistema de conocimiento científico occidental. Dicho de otro modo, que mejor satisfagan las inquietudes que guían las actividades científicas occidentales. De no ser así, se vuelven imperceptibles o simple folclore decorativo. Michael Anthony Hart deplora el desenlace como sigue:

"Así, cuando la mayoría de los profesores describen el 'mundo', describen contextos eurocéntricos e ignoran las perspectivas y entendimientos indígenas. 'Para la mayoría de los estudiantes aborígenes, la realización de su invisibilidad es similar a mirar en un lago sereno y no ver su imagen reflejada' (Henderson, 2000: 76). De hecho, el pensamiento eurocéntrico ha llegado a mediar en el mundo entero hasta el punto en que las visiones del mundo que difieren del pensamiento eurocéntrico son relegadas a la periferia, si es que se reconocen totalmente (...). Cuando se reconocen, lo más normal es que las cosmovisiones indígenas se analicen más a través de un punto de vista eurocéntrico.

Esta marginación o cegamiento de las cosmovisiones indígenas 'ha sido y sigue siendo una de las principales herramientas de la colonización' (Walker, 2004: 531). De hecho, los educadores américo-europeos, independientemente del nivel del programa educativo, preguntan a diario que las personas indígenas condesciendan o encajen en las versiones américo-europeas del mundo sin tener en cuenta sus perspectivas indígenas. La sociedad exige que alcancemos nuestros logros dentro de este modelo eurocéntrico de educación o que vivamos una vida de pobreza y dependencia de los servicio públicos como personas sin estudios, sin empleo y sin posibilidad de empleo. Así, de una forma u otra, habitualmente nos vemos obligados a validar la mitología de los colonialistas. Estamos siendo forzados a sacrificar las visiones del mundo y valores indígenas a cambio de normas externas a los objetivos culturales tradicionales (...)" (Hart, 2010: 4).

[Version original: "Thus, when most professors describe the 'world', they describe Eurocentric contexts and ignore Indigenous perspectives and understandings. "For most aboriginal 
students, the realization of their invisibility is similar to looking into a still lake and not seeing their image" (Henderson 2000: 76). Indeed, Eurocentric thought has come to mediate the entire world to the point where worldviews that differ from Eurocentric thought are relegated to the periphery, if they are acknowledged at all (...). When they are acknowledged, Indigenous worldview are analyzed most often through a Eurocentric point of view.

This marginalization or blinding of Indigenous worldviews "has taken and continues to be one of the major tools of colonization" (Walker 2004: 531). Indeed, Amer-European educators, regardless of program level, ask daily that Indigenous people acquiesce to or fit within Amer-European versions of the world while ignoring their Indigenous perspectives. Society demands that we either achieve within this Eurocentric model of education or live a life of poverty and welfare as the uneducated and unemployed or unemployable. Thus, in one way or another, we are regularly forced to validate the colonialists' mythology. We are being forced to sacrifice Indigenous worldviews and values for norms outside traditional cultural aims (...)"].

Se advierte hoy un tipo de trabajo social abierto, sensible e informado acerca de los elementos culturales locales. Esta apertura cultural llega como consecuencia de dos motivos mutuamente entrelazados. Uno es la larga historia de rotundos fracasos asociados a los programas gubernamentales de intervención social con comunidades indígenas. Otro, el objetivo institucional de responder a la presión de los movimientos sociales y el activismo cultural indígena con la receta -por un lado- de reprogramar el sistema de prestaciones sociales y -por otro- de reconducir la formación y origen de sus profesionales, con ello optimizando (econométrica y estadísticamente) la gestión o cuenta de resultados. Siendo una perspectiva emergente en las oficinas administrativas de los Estados, su mayor amplitud de reconocimiento lo está en países opulentos que miran hacia sí izando la enseña del multiculturalismo. Con todo, ocurre que su implementación, incluso en estos países, es objeto de serio criticismo desde las organizaciones sociales, culturales y comunitarias de base (Gunew y Rizvi, 1994).

En suma, no obstante los posibles efectos positivos derivados de la presente promoción cultural en el seno del trabajo social de los valores y ética concurrentes en las sociedades indígenas, los estudios al respecto asignan un perfil reformista a dicha promoción. Efectivamente, en el contexto de las sociedades indígenas contemporáneas la intervención social se caracteriza por el esfuerzo puesto en adecuarse a las circunstancias sociales y valores culturales específicos de las distintas poblaciones indias. No deja por ello de pertenecer a una corriente postrema encauzada a integrar en el sistema de conocimiento científico generalizante y universalizante occidental conjuntos específicos de partículas y fragmentos específicos de conocimiento y sociabilidad indígena. Con ello se pretende la resolución de problemas que de otro modo se perpetuarían en su irresolución [dentro de ese sistema -preliminarmente estructurado- de conocimiento universitario y práctica profesional del trabajo social]. 
En contraposición, advierte Michael Anthony Hart la emergencia de otro modelo, uno que persigue cambios y transformaciones radicales en las comunidades nativas. Denominado modelo o paradigma de investigación indígena, le impugna al trabajo social culturalmente sensible o competente recién descrito lo corto de su mira.

\section{PARADIGMAS DE INVESTIGACIÓN INDÍGENAS}

Se denominan paradigmas de investigación indígena a los modelos de investigación para el conocimiento de la realidad que sobrevienen fuera del ámbito de entendimiento e interpretación occidental de la realidad. Centrándonos en nuestro ámbito de estudio, serían las experiencias históricas y los modelos conceptuales que desde fuera del entendimiento occidental dominante se utilizan para dirimir qué es la ayuda mutua, cuándo es necesaria la intervención social y cómo se organizan las prestaciones sociales (Canda 1988; Hart, 2008; Morrissette et al., 1993). Los paradigmas de investigación indígena se insertan en lo que el ensayista argentino y profesor norteamericano Walter Mignolo ha denominado "paradigmas-otro". Los define así:

"el pensamiento crítico y utopístico que se articula en todos aquellos lugares en los cuales la expansión imperial/colonial le negó la posibilidad de razón, de pensamiento y de pensar el futuro. Es 'paradigma otro' en última instancia porque ya no puede reducirse a un 'paradigma maestro', a un 'paradigma nuevo' que se autopresente como la 'nueva' verdad. La hegemonía de un 'paradigma otro' será utopisticamente, la hegemonía de la diversidad, esto es, 'de la diversidad como proyecto universal (...) y no ya un 'nuevo universal abstracto' " (Mignolo, 2003: 20).

Intelectuales indígenas originarios de países históricamente anejos a la órbita cultural colonial anglosajona como Michael Anthony Hart afirman que cualquier discusión sobre la posible paridad o discrepancia entre el pensamiento científico occidental y el sistema o sistemas de conocimiento e investigación indígena de la realidad debe formularse a partir del examen de la noción cosmovisión, entendiendo por ésta la manera de ver, interpretar y actuar sobre el mundo según la experiencia existencial e histórica de los individuos y sus colectividades (Hart, 2010: 2).

Según este punto de partida, los sistemas de investigación/conocimiento indígena y los sistemas de investigación/conocimiento occidental son paradigmas de interpretación del mundo, y de su orden de funcionamiento, arraigados en marcos mentales que han sido urdidos con hilos lógico-conceptuales analógicamente equivalentes. Sin embargo, el hecho de que han existido y existen relaciones -nacionales e internacionales- de poder continuado entre sociedades y culturas ha creado, diseminado y reproducido, entre otros asuntos, imágenes positivas y negativas sobre los distintos tipos de investigación, conocimiento y acción sociocultural con que las distintas sociedades y culturas operan sobre el mundo en que se desenvuelven. 
Pese al ideal de universalidad que gobierna el pensamiento científico occidental, en él reina la disparidad. También en el contorno de las cosmovisiones indígenas se observa variedad de conjunto e incongruencias dentro de cada sistema, lo que no impide ver en ellas una base común excepcional en su género, a saber: su conexión con el conjunto del medio ambiente, e igualmente con cada uno de sus componentes particulares, lo es en términos de reciprocidad relacional. Esta característica de sus cosmovisiones las distingue sobremanera de las occidentales, donde lo normal es que las relaciones hombre/naturaleza se encaucen -incluso entre los campesinos- desde fuera del orden de intercambios que regulan los ecosistemas. Y es que entre los tipos de cosmovisión occidental sobresale el que ideológicamente tiende a situar al género humano (y dentro de este al segmento varón) por encima de la totalidad de haces de relaciones propias de los ecosistemas, operando sobre estas desde posiciones de dominación externa.

Con el fin de ilustrar con mayor grado de exigencia este contraste entre cosmovisiones, Michael Anthony Hart ha recurrido al trabajo de varios profesores universitarios indígenas norteamericanos, de los que en este artículo voy a nombrar tres: Leanne Betasamosake Simpson, Eva Marie Garroutte y Shawn Wilson. De Leanne Betasamosake Simpson, artista, académica y activista de las primeras naciones de Canadá, recoge así:

"Leanne Simpson (2000) esbozó siete principios de cosmovisiones indígenas. En primer lugar, el conocimiento es holístico, cíclico, y depende de las relaciones y conexiones para con los seres vivos y no vivos y demás entes. En segundo lugar, hay muchas verdades, y estas verdades dependen de las experiencias individuales. En tercer lugar, todo está vivo. En cuarto lugar, todas las cosas son iguales. En quinto lugar, la tierra es sagrada. En sexto lugar, la relación entre las personas y el mundo espiritual es importante. En séptimo lugar, los seres humanos son menos importantes en el mundo" (Hart, 2010: 3).

[Versión original: "Leanne Simpson (2000) outlined seven principles of Indigenous worldviews. First, knowledge is holistic, cyclic, and dependent upon relationships and connections to living and non-living beings and entities. Second, there are many truths, and these truths are dependent upon individual experiences. Third, everything is alive. Fourth, all things are equal. Fifth, the land is sacred. Sixth, the relationships between people and the spiritual world is important. Seventh, human beings are least important in the world"]. De Eva Marie Garroutte (2003), afín a la nación Cheroquee, acoge la noción de indigenismo radical, consistente en el proceso de búsqueda de una ciencia general indígena reconocida en igual de condiciones que otros tipos de conocimiento de representación de la realidad circundante. Y de Shawn Wilson, indio cree de Canadá y profesor en la universidad australiana de Sydney, toma su conceptualización del proceso cognitivo que estructura la producción de conocimiento indígena. Arropado en el trabajo de estas tres personas -Leanne Betasamosake Simpson, Eva Marie Garroutte y Shawn Wilson- Michael Anthony Hart defiende la existencia de un paradigma de investigación específicamente indígena, que demarca como sigue. 
Primero, su dimensión ontológica. Las comunidades indígenas conceptualizan esferas espirituales de relaciones en conexión con esferas de relaciones físicas. Se materializan tanto simbólica como materialmente siguiendo pautas de reciprocidad que se canalizan y estructuran en ceremoniales y reuniones rituales específicas. Segundo, su dimensión epistemológica. Hay un sistema de pensamiento indígena con su propia lógica de conocimiento. Esta pertenece al ámbito de las experiencias introspectivas intensas que ocurren en el interior del nexo comunicativo operante entre las distintas esferas de relaciones mutuas que mantienen los seres vivos, los espíritus y los objetos físicos. Por su parte, los ensayos de introspección se gestan en laboratorios y centros de experimentación de conocimiento concretos; a saber: en los rituales y las ceremonias, donde ensueño, alteración de la conciencia, visión, meditación e invocación conducen dicho proceso de introspección. El transcurso de conservación, enseñanza y transmisión intergeneracional de los conocimientos así alcanzados queda sancionado en las situaciones colectivas de narración oral. Consiguientemente -explica Hart- crear, experimentar y transmitir conocimiento en el mundo indígena consiste en poner en marcha una praxis colectiva de comunicación.

Tercero, su dimensión metodológica. Esta descansa sobre la idea de reciprocidad de la vida, lo que implica una rendición de cuentas relacional y colectiva. El énfasis recae en la aplicación práctica del conocimiento adquirido dentro del sistema de reciprocidad entre humanos, espíritus y objetos físicos. Y cuarto, su dimensión ética. En el paradigma de investigación indígena, esta está vinculada a la comunidad. Puesto que la investigación indígena se plantea para el beneficio de la comunidad indígena, su realización está supedita al control comunitario del proceso de investigación y generación de conocimiento. El paradigma indígena evita la intrusión. Pone el acento en la percepción de las emociones. Sus buenas maneras consisten en escuchar, oír, ver y mirar con respeto, con silencio, con atención, reflexivamente y sin juzgar. Privilegia el pálpito del corazón que mueve las palabras y las acciones que ocurren alrededor. La ética en el paradigma de investigación indígena consiste en el reconocimiento subjetivo del proceso creativo de la persona que crea conocimiento y lo transmite en un contexto colectivo (Hart, 2010).

\section{A MODO DE REFLEXIÓN FINAL}

Según hemos expuesto arriba el criterio indígena de investigación y conocimiento de la realidad contrasta desmesuradamente con el criterio científico occidental. Nos hemos preguntado hasta qué punto los conocimientos indígenas forman parte del cuerpo de saber universitario relacionado con la enseñanza del trabajo social y su práctica profesional con comunidades indígenas.

La forja del trabajo social, así como la del sistema político institucional organizativo, que lo acondiciona e impulsa, es un legado intelectual y profesional específico del artificio 
modernista occidental. Su enseñanza e investigación universitarias forman parte del sistema de conocimiento científico modernista occidental, poco o nada interesado en los sistemas de conocimiento propios de las sociedades y culturas indígenas.

El conocimiento social científico procedente de la institución universitaria funciona a modo de dispositivo comunicativo con el que escenificar un imaginario particular, el de la modernidad occidental y su máquina productora de conocimientos científicos. Las imágenes conceptuales que se procesan, transmiten y propagan desdeloscentros superiores de investigación y enseñanza universitaria en términos de conocimiento científico lucen las inscripciones de "saber verdadero" y "saber autorizado". Su omnipresencia ha acarreado un resultado perverso: los relatos explicativos contemporáneos sobre la desdicha y el sufrimiento indígena omiten el grado de responsabilidad que en rigor le corresponde al núcleo económico e ideológico originariamente causante de esa desdicha y sufrimiento: a saber, la modernidad europea.

El discurso occidental de la verdad científica evita un asunto ético y político capital: el ensamblaje de las imágenes conceptuales con que la modernidad occidental se define a sí misma es parte consustancial de otro ensamblaje de imágenes conceptuales: el que explica la colonialidad indígena y su status quo subalterno. La universidad es una institución dedicada a la construcción de saberes universales y objetivos. Su misión consiste en forjar conocimientos veraces, saberes científicamente autorizados. Pero lleva un envés: legitima el expolio, expropiación, segregación, arrinconamiento, rechazo o erradicación de aquellos conocimientos y saberes no autorizados.

Stricto sensu, la propuesta de articular programas universitarios de trabajo social comunitario indígena es contradictoria en sí misma. Se trata de la paradoja de crear un título universitario, formal y científicamente autorizado, aplicado al desarrollo e implementación de saberes no autorizados, postergados y repudiados. Su ejecución, sin duda, comprometería la idea occidental de conocimiento científico, y esquivaría la de trabajo social. 


\section{BIBLIOGRAFÍA}

Abatena, Hailu (1995) "The importance of community self-help activities in promoting social development". Journal of Social Development in Africa 10, pp. 5-24.

Álvarez Molinero, Natalia; Oliva Martínez, Daniel y Zúñiga García-Falcés, Nieves (eds.) (2009) Declaración sobre los derechos de los pueblos indígenas. Madrid: Libros de la Catarata.

Anaya, James (1996) Indigenous peoples in international law. Oxford: Oxford University Press.

Asad, Talad (ed.) (1973) Anthropology and the colonial encounter. New York: Humanities Press.

Ashcroft, Bill; Griffiths, Gareth y Tiffin, Hellen (eds.) (1995) The postcolonial studies reader. Londres: Routledge.

Austin-Broos, Diane J. (ed.) (1987) Creating culture. Sydney: Allen y Unwin.

Bartoli, Laura (2002) Antropología aplicada: historia y perspectivas desde América Latina. Quito: Abya-Yala.

Bonfil, Guillermo (1987) México profundo, una civilización negada. México D.C.: Grijalbo.

Canda, Edward R. (1988) "Conceptualising spirituality for social work: insights from diverse perspectives”. Social Casework 69, pp. 238-247.

Caride, José Antonio y Vieites, Manuel F. (coord.) (2006) De la educación social a la animación teatral. Gijón: Ediciones Trea.

Clark, Natalie et al. (2012) “Indigenous social work field education: Melq’ilwiye’ coming together towards reconciliation”. Native Social Work Journal 8, pp. 105-127.

Cohen, Anthony (1985) The symbolic construction of community. Londres: Routledge.

Cole, John W. (1977) "Anthropology comes part-way home: community studies in Europe”. Annual Review of Anthropology 6, pp. 349-378.

Craig, Gary y Mayo, Marjorie (eds.) (1995) Community empowerment: a reader in participation and development. Londres: Zed Books.

Delaney, Roger; Brownlee, Keith y Zaft, Kim (eds.) (1999) Social work practice with rural and northern peoples. Thunder Bay. Ontario: Center for Northern Studies.

Dixon, John y Scheurell, Robert P. (eds.) (1995) Social Welfare with indigenous peoples. Londres: Routledge.

Dominelli, Lena (ed.) (2007) Revitalizing communities in a globalizing world. Aldershot: Ashgate. 
During, Simon (ed.) (1993) The cultural studies reader. Routledge: Londres.

Escobar, Arturo (1995) Encountering development: the making and unmaking of the Third World. Princeton: Princeton University Press.

Fernández de Larrinoa, Kepa (2014) Del delito sociocultural y su resolución sociopolítica. Certeza: Zaragoza.

Ferro, Marc (2005) El libro negro del colonialismo (siglos XVI al XIX: del exterminio al arrepentimiento). Madrid: La Esfera de los Libros.

Garroutte, Eva M. (2003) Real Indians: identity and the survival of Native America. Berkeley: University of California Press.

Giroux, Henry A. (1997) Cruzando límites: trabajadores culturales, políticas educativas. Barcelona: Paidós.

Giroux, Henry A. (2001) Cultura, política y práctica educativa. Graò: Barcelona.

Gray, Mell; Coates, John y Yellow Bird, Michael (eds.) (2008) Indigenous social work around the world: towards culturally relevant education and practice. Aldershot: Ashgate.

Gray Mell; Coates, John; Yellow Bird, Michael y Hetherington, Tiani. (eds.) (2013) Decolonizing social work. Aldershot: Ashgate.

Gunew, Sneja y Rizvi, Fazal (eds.) (1994) Culture, difference and the arts. St. Leonards, New South Wales: Allen and Unwin.

Hall, Stuart (1997) Representation: cultural representations and signifying practices. Sage: London y California: Thousand Oaks.

Harrison, Barbara (2001) Collaborative programs in indigenous communities: from fieldwork to practice. Oxford: Altamira Press.

Hart, Michael Anthony (2008) "Critical reflections on an aboriginal approach to helping". En Mel Gray, John Coates y Michael Yellow Bird (eds.) Indigenous social work around the world: towards culturally relevant education and practice. Aldershot: Ashgate, pp. 129139.

Hart, Michael Anthony (2010) "Indigenous worldviews, knowledges, and research: the development of an indigenous research paradigm". Journal of Indigenous Voices in Social Work 1, pp 1-16.

Harper, Kenn (1986) Give me my father's body: the life of Minik, the New York Eskimo. Hanover: Steerforth Press.

Henderson, J. Sajek Y. (2000) "Challenges of respecting Indigenous world views in a Eurocentric education”. En R. Nail (ed.) Voice of the drum: Indigenous education and culture. Brandon, Manitoba, Canadá: Kingfisher Publication, pp. 59-80 
Hendry, Joy (2014) Science and sustainability: learning from Indigenous wisdom. Londres: Palgrave.

Hobart, Mark ed. (1993) An anthropological critique of development: the growth of ignorance. Londres: Routledge.

hooks, bell (2000) Feminism is for everybody: passionate politics. Cambridge, MA: South End Press.

Huntington, Henry P. (2000) "Using traditional ecological knowledge in science: methods and applications". Ecological Applications 10 (5), pp. 1270-1274.

James, P.; Nadarajah, Y.; Haive, K. y Stead V. (2012) Sustainable communities, sustainable development: other paths for Papua New Guinea. Honolulu: University of Hawai'i Press. Jameson, Fredric (1991) Postmodernism, or the cultural logic of late capitalism. Durham: Duke University Press.

Kothari, Miloon (ed.) (1999) Development and social action. Oxford: Oxfam.

Kuper, Adam (ed.) (1992) Conceptualizing society. Londres: Routledge.

Lévi-Strauss, Claude (1996) Raza e historia. Raza y cultura. Madrid: Cátedra.

Luszczynska, Ana M. (2012) The ethics of community: Nancy, Derrida, Morrison and Menendez. Londres: Continuum.

Marcus, George y Fischer, Michael (1986) Anthropology as cultural critique. Chicago: University of Chicago Press.

McDonald, James H. (ed.) (2002) The applied anthropology reader. Boston: Allyn \& Bacon.

McTaggart, Robin (ed.) (1997) Participatory action research: international contexts and consequences. Nueva York: State University of New York Press.

Menzies, Charles R. (ed.) (2006) Traditional Ecological Knowledge and natural resources management. Lincon: University of Nebraska Press.

Mezzadra, Sandro (ed.) (2008) Estudios postcoloniales: ensayos fundamentales. Madrid: Mapas.

Midgley, James (1981) Professional imperialism: social work in the Third World. Londres: Heinemann Educational Books.

Mignolo, Walter (2003) Historias locales / diseños globales: colonialidad, conocimientos subalternos y pensamiento fronterizo. Madrid: Akal.

Mignolo, Walter (2007) "Coloniality of power and de-colonial thinking". Cultural Studies 21, pp. 155-167. 
Morrissette, Vern; McKenzie, Bradley y Morrissette, Larry. (1993) “Towards an aboriginal model of social work practice: cultural knowledge and traditional practices". Canadian Social Work Review 10, pp. 91-108.

Mum Shirl, con la colaboración de Bobbi Sykes (1992). Mum Shirl: an autobiography. Melborune: Mammoth.

Munford, Robyn y Sanders, Jackie (2010) "Embracing the diversity of practice: indigenous knowledge and mainstream social work practice". Journal of Social Work Practice 25(1), pp. 63-77.

Payne, Malcolm (1991) "Social and community development". En Malcolm Payne (ed.) Modern social work theory. Nueva York: Palgrave, pp. 198-213.

Peat, F. David (2012) “Tradicional knowledge and Western science". En Joy Hendry and Laara Fitznor (eds.). Anthropologists, Indigenous scholars and the research endeavour: seeking bridges towards mutual respect. Londres: Routlege, pp. 118-127.

Pink, Sarah (ed.) (2006) Applications of anthropology: professional anthropology in the twenty-first century. Oxford: Berhahn Books.

Quijano, Anibal (2000) "Colonialidad del poder y clasificación social”. Journal of World System Research 6, pp. 342-386.

Rahnema, Majid y Bawtree, Victoria (ed.) (1997) The post-development reader. Londres: Zed Books.

Said, Edward W. (1993) Culture and Imperialism. Londres: Chatto y Windus.

Sánchez Sánchez, Rafael (coord.) (2003) Animación sociocultural y desarrollo rural. Zaragoza: CEDDAR, Centro de Estudios sobre Despoblación y Desarrollo de Áreas Rurales.

Saugestad, Sidsel (2001) The inconvenient indigenous: remote area development in Botswana, Donor Assistance, and the First People of the Kalahari. Uppsala: The Nordic Africa Institute.

Sillitoe, Paul; Bicker, Alan y Pottier, Johan (2002) Participating in development: approaches to indigenous knowledge. London: Routledge, ASA Monographs 39.

Simpson, Leanne B. (2000) "Anishinaabe ways of knowning". En J. Oakes, R. Riew, S. Koolage, L. Simpson y N. Schuster (eds.) Aboriginal health, identity and resources. Winnipeg, Manitoba: Native Studies Press, pp. 165-185.

Smith, Linda T. (1999) Decolonizing methodologies: research and indigenous peoples. Londres: Zed Books. 
Stocking, George W. Jr. (ed.) (1974) "Part X: Anthropology and society”. En Franz Boas A Franz Boas reader: the shaping of American anthropology, 1883-1911. Chicago: The University of Chicago Press, pp. 307-340.

Tacchi, Jo A.; Slater, Don y Hearn, Gregory N. (2003) Ethnographic action research. New Delhi: Unesco.

Taylor, Samuel H. y Roberts, Robert W. (eds.) (1985) Theory and practice of community social work. Nueva York: Columbia University Press.

Terdiman, Richard (1985) Discourse / counter-discourse: the theory and practice of symbolic resistance in nineteenth-century France. Ithaca: Cornell University Press.

Truell, Rory (2012) The Global Agenda for Social Work and Social Development: extending the influence of social work. ISSW.

Valcárcel-Resalt, Germán (ed.) (1997) Cultura y desarrollo. Madrid: Fundación Navapalos y Universidad de Valladolid.

Van Erven, Eugene ed. (2013) Community, art power. Rotterdam: Rotterdams Wijktheater. Verhelst, Thierry y Tyndale, Wendy (eds.) (2002) Development and culture. Oxford: Oxfam.

Walker, Polly O. (2004) “Decolonizing conflict resolution”. American Indian Quarterly 28 (3-4), pp. 527-549.

Weaver, Hilary N. (1999) "Indigenous people and the social work profession: defining culturally competent services”. Social Work 44, pp. 217-225.

Wilson, Shawn (2001) “What is Indigenous research metodology?". Canadian Journal of Native Education 25(1), pp. 175-179.

Wolf, Eric (1987) Europa y la gente sin historia. México D.F.: Fondo de Cultura Económica. Wright, Susan (1992) "Image and analysis: new directions in community studies". En B. Short (ed.) The English rural community: image and analysis. Cambridge: Cambridge University Press, pp. 195-217.

Wuthnow, Robert; Hunter, James Davison; Bergersen, Albert y Kurzweil, Edith (1984) Cultural analysis: phenomenology, cultural anthropology, neo-structuralism, critical theory. Londres: Routledge y Kegan Paul. 